\title{
In vitro biocompatibility testing of polymeric nanofiber scaffolds: fine-tuning for a better prediction of the in vivo behaviour
}

\begin{abstract}
Biomaterial research efforts focus on the development of biomaterials that mimic the natural extracellular environment. In addition, different strategies are applied to render materials for blood-contacting devices nonthrombogenic through surface modifications that would suppress activation of platelets, coagulation and the complement system. A confluent thin layer of endothelial cells lines all blood vessels and produces factors responsible for inhibition of coagulation, thrombosis and neointimal hyperplasia. Thus, the ability to rapidly form a healthy endothelium upon implantation represents a desired property of biomaterials used for cardiovascular devices. In this study we used advanced in vitro methods to investigate the biocompatibility of a biodegradable and a permanent electrospun nanofiber fabric, poly-L-lactic acid and polycarbonate-based silicone elastomer respectively, with the focus on endothelialization and hemocompatibility.
\end{abstract}

Keywords: nonwovens, electrospinning, PLLA, TSPCU, endothelialization, biocompatibility, hemocompatibility.

https://doi.org/10.1515/cdbme-2021-2158

\section{Introduction}

Cardiovascular diseases are the number one cause of death worldwide [1]. Many efforts on the field of biomedical engineering focus on the development of blood-contacting devices with the mechanical compliance and biological

\footnotetext{
*Corresponding author: Valeria Khaimov: Institute for ImplantatTechnology and Biomaterials e.V., Friedrich-BarnewitzStrasse 4, 18119 Rostock-Warnemünde, Germany, e-mail: valeria.khaimov@uni-rostock.de

Bastian-Jesper Klußmann-Fricke, Stefan Siewert, Klaus-Peter Schmitz: ImplantatTechnology and Biomaterials e.V., 18119 Rostock-Warnemünde, Germany Sabine IIIner, Klaus-Peter Schmitz: Institute for Biomedical Engineering, Rostock University Medical Center, RostockWarnemünde, Germany
}

properties similar to the native environment at the tissueimplant interface. Such materials should minimize the activation of platelets, coagulation cascade and tissue overgrowth - well studied mechanisms behind the thrombus formation and neointimal hyperplasia leading to implant failure [2]. A confluent thin layer of endothelial cells lines all blood vessels and produces factors responsible for inhibition of thrombosis and neointimal hyperplasia [3-5]. Thus, the ability to rapidly form a healthy endothelium upon implantation represents a desired property of biomaterials used for cardiovascular devices.

Electrospinning is a versatile process for fabrication of polymeric biomaterials with unique physicochemical properties that allow for an optimal integration of the implant into the inflammatory tissue environment [3].

Biocompatibility testing according to DIN EN ISO 10993 evaluates the safety of a medical device in its final form prior to initiation of clinical studies. The choice of the right polymer for the biomaterial fabrication and its processing to a final biomaterial are crucial steps during the development of new medical devices and require extensive experience in biocompatibility testing. Thus, in vitro biocompatibility testing of the sole biomaterial during the early development stages is extremely important and should use cell culture models that resemble the biological environment at the implantation site. In addition, blood contacting devices need to be evaluated with respect to hemocompatibility. Although different methods exist to address blood-implant interactions, the main obstacle is the lack of standardization on this field [6].

In this study we used advanced in vitro methods to investigate the biocompatibility of a fully biodegradable [7] and permanent electrospun nanofiber fabric, poly-L-lactic acid (PLLA) and polycarbonate-based silicone elastomer (TSPCU) respectively, with the focus on endothelialization and hemocompatibility. 


\section{Methods}

\subsection{Scaffold fabrication and treatment}

Homogenous polymer solutions of $4 \mathrm{wt} \%$ PLLA (RESOMER L210, Evonik, Germany) and $7.5 \mathrm{wt} \%$ TSPCU (Chronosil 55D, Advansource, USA) were obtained by dissolving commercially available polymers in a mixture of trifluorethanol/chloroform (4/1) and dimethylformamide/ trifluorethanol/chloroform (1/1/8), respectively. Scaffolds of $100 \times 200 \mathrm{~mm}$ in size were generated by dynamic electrospinning at 4SPIN C4S LAB2 (Contipro, Czech Republic) at room temperature. Fiber structure and diameter were assessed via scanning electron microscopy (SEM, Quanta 250 FEG, FEI Company, USA).

\subsection{Cell culture}

All media components were purchased from PAN Biotech (Germany). Human plasma was from Affinity Biologicals Inc (Canada). Human endothelial cells EA.hy926 (ATCC) were maintained in Dulbecco's Modified Eagle's Medium/10\% fetal calf serum (FCS))/antibiotics (pen/strep) at $37^{\circ} \mathrm{C}, 5 \%$ $\mathrm{CO}_{2}$ under humidified atmosphere. For experiments $10^{4}$ cells per well were used. For cytotoxicity testing, material extracts were prepared according to DIN EN ISO 10993 and used for cell treatment, followed by viability testing (CellQuantiBlue, BioAssaySystems, USA). Data was normalized to cells grown on tissue culture-treated polystyrene (TCpolystyrene).

\subsection{Microscopy and image analysis}

For the vacuum treatment scaffolds were covered with water and briefly incubated at 20 mbar (Memmert, Germany). Vacuum treated or untreated samples were incubated with FCS, human plasma or serum at $37^{\circ} \mathrm{C}$ overnight. Scaffolds were rinsed with PBS and incubated with $10^{4}$ cells/well for $48 \mathrm{~h}$. Cells were rinsed with phosphate buffered saline (PBS) and fixed in formafix (PathoMed. Logistik GmbH, Germany) for $10 \mathrm{~min}$. Cells were washed with PBS and permeabilized with $0.5 \%$ Triton X-100/PBS. Nuclei and actin were stained with NucBlue Fixed Cell Stain and ActinRed555 ReadyProbes (Invitrogen, USA) followed by confocal microscopy (Olympus FV-1000, Japan) at 200x. Initial image processing (background subtraction, contrast adjustment, maximum intensity projections) of the acquired z-stacks was performed with Fiji (NIH, USA). Quantitative analysis of at least three representative images for each sample was performed with CellProfiler and cellular parameters such as cell morphology and area covered by cells have been measured.

\subsection{Hemocompatibility testing}

Platelet rich plasma (PRP) was isolated from fresh blood of healthy d. $13 \mathrm{~mm}$ polymer punches were incubated with 1.5 $\mathrm{ml}$ of PRP in a 24 well plate at room temperature. TCpolystyrene was used as reference material. At time point 0 and $1 \mathrm{~h} 1 \mathrm{ml}$ of each sample was transferred into CTAD tubes (Sarstedt, Germany) on ice. PF4 release was assayed by ELISA (Asserachrom, France). For SEM analysis scaffolds were fixed with $2.5 \%$ buffered glutaraldehyde, followed by dehydration in an ascending alcohol series and hexamethyldisilazane. Samples were mounted on carriers and sputter coated with gold prior to measurement at Quanta FEG 250 (FEI Company, USA). For the complement activation assay scaffolds were incubated for $2 \mathrm{~h}$ with human normal serum (Quidel, USA) in an overhead mixer at $37^{\circ} \mathrm{C}$. Supernatants were harvested by the addition of the specimen stabilizer (Quidel) and stored at $-20^{\circ} \mathrm{C}$. Formation of the terminal complement complex was assayed by enzyme immunoassay (MicroVue SC5b-9, Quidel).

\section{Results}

\subsection{Fiber diameter and cytotoxicity}

SEM analysis of polymeric nanofibrous scaffolds fabricated either from PLLA or TSPCU revealed fiber diameters mainly ranging from 500-1000 nm for both polymers. (Figure 1, Table 1). Next, nonwovens were tested for cytotoxicity according to DIN EN ISO 10993 (Figure 2). While none of the nanofiber scaffolds tested showed a negative impact on cell viability, cells treated with TSPCU extracts showed reproducible increased metabolic activity as compared to TCpolystyrene with values $\sim 130 \%$.

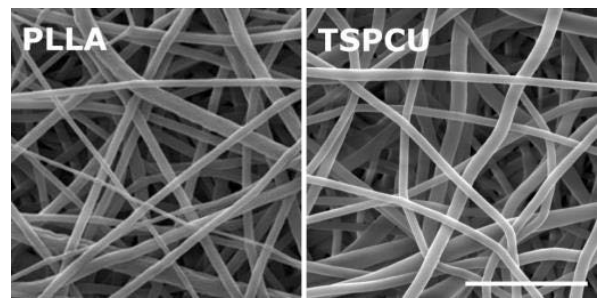

Figure 1: SEM analysis of fabricated scaffolds. Scale bar: $10 \mu \mathrm{m}$. 
Table 1: Fiber diameters of PLLA and PCU- Si nonwovens

\begin{tabular}{ccc}
\hline Sample & $\begin{array}{c}\text { Polymer } \\
\text { concentration [wt\%] }\end{array}$ & $\begin{array}{c}\text { Fiber diameter } \\
\text { [nm] }\end{array}$ \\
\hline PLLA & 4 & $500-900$ \\
TSPCU & 7,5 & $600-900$ \\
\hline
\end{tabular}

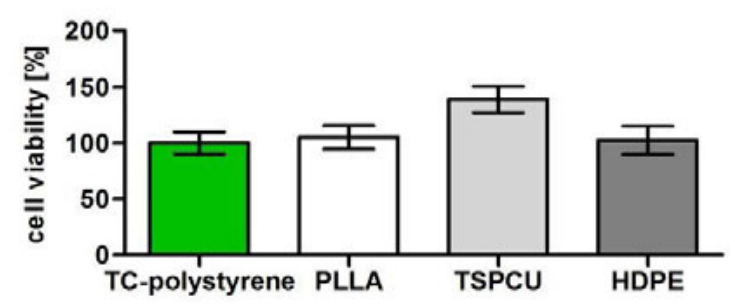

Figure 2: Viability of endothelial cells on TC-polystyrene (100\%) or material extracts. HDPE: high density polyethylene, biocompatible reference material (mean $\pm S D, n=3$ ).

\section{2 in vitro assessment of the endothelialization of nonwovens}

OIn a typical approach to the assessment of the endothelialization potential, cells are seeded on top of a scaffold. However, cell morphology is frequently affected and cell adhesion is poor due to the hydrophobic nature of polymers and the air trapped between the nanofibers $[8,9]$. In addition, this in vitro approach only partly resembles the situation in vivo, where proteins from blood are adsorbed at the biomaterial surface immediately upon implantation, also known as the Vroman effect [10]. In order to challenge and further improve the classical in vitro system, nonwovens were subjected to low vacuum and/or preconditioned with FCS, human plasma or human serum overnight at $37^{\circ} \mathrm{C}$ prior to cell seeding. Microscopic analysis revealed that some samples showed huge differences in cell morphology (round vs. elongated) and cell density at the centre and border of the polymeric discs. Despite this fact, area covered by cells and cell morphology was bigger when nonwovens were preconditioned with plasma (Figure 3). In addition, quantitative analysis of representative images showed that data sets, where no vacuum was applied, had higher fluctuations with respect to biomaterial-cell-interactions as indicated by standard deviations (Figure 4). The highest value for the occupied area was in the sample treated by vacuum and plasma ( $\sim 60 \%)$. Quantification of round cells in the sample resulted in the lowest value for the plasma treated scaffolds $(\sim 10 \%)$ and the highest for no treatment $(\sim 45 \%)$ or treatment with water $(\sim 30-60 \%)$, respectively (data not shown). Also, preconditioning with FCS seems to support cell adhesion to scaffolds (Figure 3,4). Noteworthy, no obvious differences were observed between cell adhesion to PLLA and TSPCU nonwovens.

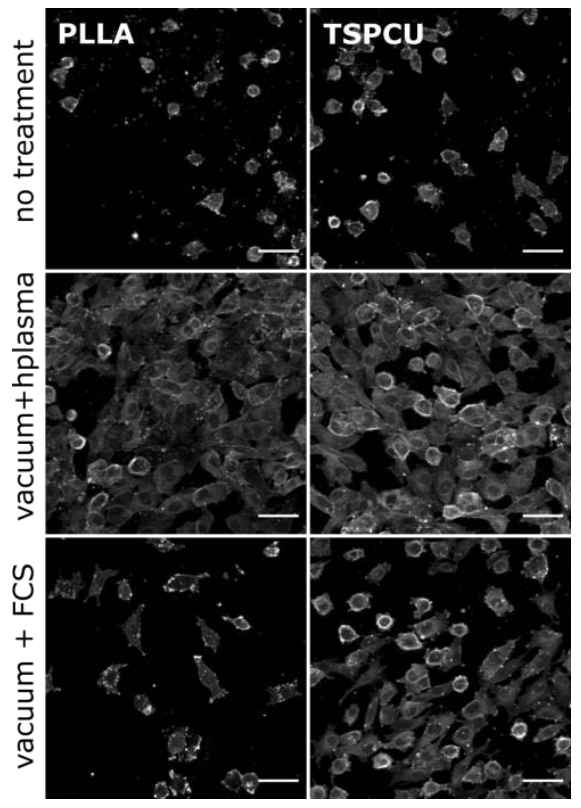

Figure 3: Cell morphology and scaffold coverage by endothelial cells. Cytoskeleton (actin) is shown in grays (scale bar $50 \mu \mathrm{m}$ ).

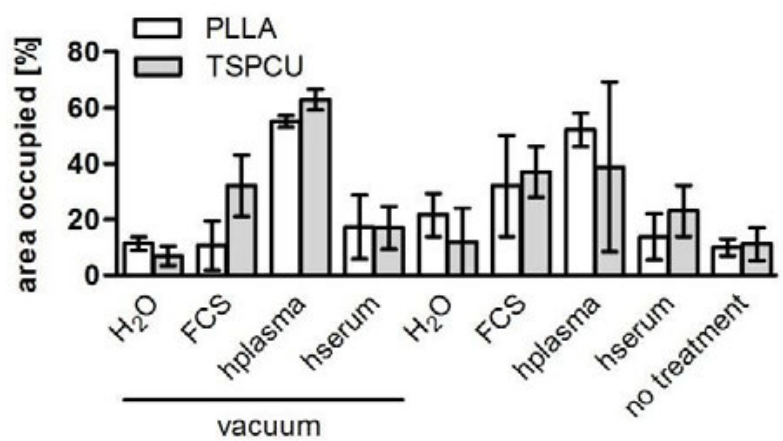

Figure 4: Quantitative image analysis of the scaffold area covered by cells upon treatment (mean $\pm S D, n=3$ ).

\subsection{Hemocompatiblity testing}

Biomaterials used for blood contacting medical devices should prevent thrombus formation and inflammation. Thus, in the next step we evaluated the blood-material interactions with respect to platelet and complement activation. First, platelet rich plasma was incubated with nonwovens or TCpolystyrene under static conditions. SEM analysis showed greater coverage of PLLA-nanofibers as compared to TSPCU indicating stronger platelet activation at the PLLA surface (Figure 5). Slightly more platelet factor 4 (PF4) was released from platelets when incubated with PLLA supporting this finding (Figure 6). Finally we tested the activation of the complement system in an in vitro system based on standardized human serum. Importantly, neither PLLA nor 
TSPCU increased complement activation as compared serum in absence of scaffolds (Figure 7).
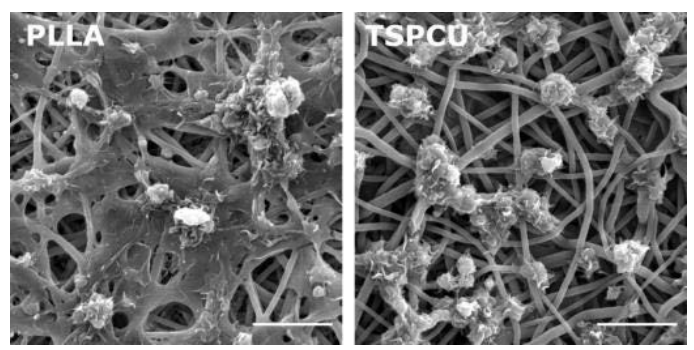

Figure 5: SEM analysis of nonwovens upon contact with plateletrich plasma (scale bar: $10 \mu \mathrm{m}$ ).

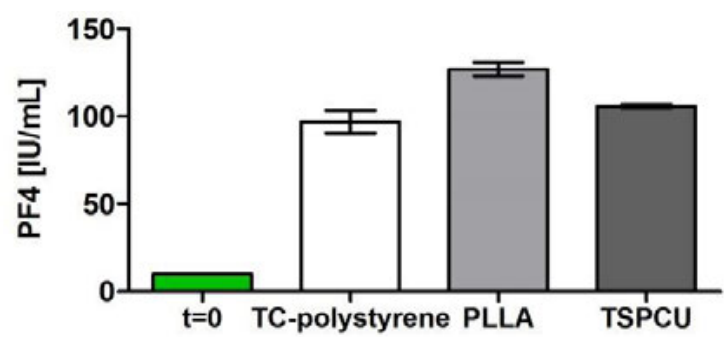

Figure 6: PF4 release upon platelet-scaffold interaction for $1 \mathrm{~h}$ at room temperature (mean $\pm S D, n=2$ ).

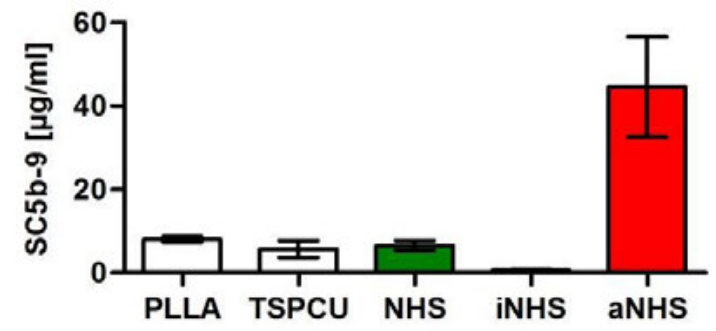

Figure 7: Complement activation upon contact of serum with scaffolds (mean $\pm \mathrm{SD}, \mathrm{n}=2$ ). (NHS: serum without biomaterial, iNHS: complement inhibition by EDTA, aNHS: strong complement activation by zymosan).

\section{Conclusions}

Formation of a functional endothelial layer is crucial for the performance of blood-contacting implants. In this study, a biodegradable and a permanent material was successfully fabricated by electrospinning and tested for biocompatibility. Both biomaterials tested here (PLLA and TSPCU) were not cytotoxic and did not activate the complement system. Although cells were rounded on untreated materials, cell morphology improved dramatically upon treatment with plasma. While platelet activation by nonvowens was slightly more pronounced for PLLA as compared to TSPCU, a clearly bigger area of PLLA scaffolds was covered by platelets upon contact with PRP. Our data shows that finetuning (i.e. plasma/vacuum treatment) of commonly used in vitro testing systems can have a huge impact on the performance of biomaterials. Further work needs to be done in order to reveal the mechanisms behind the enhanced endothelialization of biomaterials upon plasma treatment and its relevance for the assessment of biocompatibility according to DIN EN 10993. Moreover, the in vitro complement activation assay presented here provides a valuable and robust testing system for the initial screening and optimization of biomaterials with respect to hemocompatibility.

\section{Author Statement}

Research funding: Partial financial support by the Federal Ministry of Education and Research (BMBF) within RESPONSE "Partnership for Innovation in Implant Technology" and by the European Social Fund (ESF) within the excellence research program of the state MecklenburgVorpommern is gratefully acknowledged. Authors state no conflict of interest. Ethical approval: The study was conducted under ethics approval of Rostock University Medical Center. Informed consent: Informed consent has been obtained from all individuals included in this study. Acknowledgement: The authors acknowledge technical assistance of Katja Hahn and Martina Nerger.

\section{References}

[1] https://www.who.int/health-topics/cardiovascular-diseases/, accessed 1 July 2021.

[2] Li j, Zhang K, Huang N. Engineering Cardiovascular Implant Surfaces to Create a Vascular Endothelial Growth Microenvironment. Biotechnol. J. 2017;12:1600401.

[3] Bruna BBJ, Wakabayashi N, Oyama K, Kamiya H, Braghirolli DI, Pranke P. Vascular Tissue Engineering: Polymers and Methodologies for Small Caliber Vascular Grafts. Frontiers in Cardiovascular Medicine 2021;7:376.

[4] Ren X, Feng Y, Guo J, Wang H, Li Q, Yang J, Hao X, Lv J, Ma N, Li W. Surface modification and endothelialization of biomaterials as potential scaffolds for vascular tissue engineering applications. Chem Soc Rev. 2015;44(15):56805742.

[5] Strohbach A, Busch R. Polymers for Cardiovascular Stent Coatings. Int J Polym Sci. 2015;2:1-11.

[6] Sperling C, Maitz MF, Werner C. Test methods for hemocompatibility of biomaterials. Hemocompatibility of Biomaterials for Clinical Applications. 2018:77-104.

[7] Bergstrom, JS; Hayman D. An Overview of Mechanical Properties and Material Modeling of Polylactide (PLA) for Medical Applications. Ann. Biomed. Eng. 2016, 44, 330-340.

[8] Matschegewski C, Matthies JB, Grabow N und Schmitz KP. Cell adhesion and viability of human endothelial cells on electrospun polymer scaffolds. Current Directions in Biomedical Engineering 2016;2(1):11-14.

[9] Szewczyk PK, Ura DP, Metwally S, Knapczyk-Korczak J, Gajek M, Marzec MM, Bernasik A, Stachewicz U. Roughness and Fiber Fraction Dominated Wetting of Electrospun FiberBased Porous Meshes. Polymers (Basel). 2018; 27;11(1):34.

[10] Christo SN, Diener KR, Bachhuka A, Vasilev K, Hayball JD. Innate Immunity and Biomaterials at the Nexus: Friends or Foes, BioMed Research International, 2015: 342304. 